\title{
Ethnologies
}

\section{Médiation culturelle pour la sauvegarde et la valorisation du patrimoine tunisien \\ Des initiatives citoyennes}

\section{Selma Zaiane-Ghalia}

Volume 38, numéro 1-2, 2016

Créativité et médiation en tourisme et en patrimoine

Creativity and mediation in tourism and heritage

URI : https://id.erudit.org/iderudit/1041590ar

DOI : https://doi.org/10.7202/1041590ar

Aller au sommaire du numéro

\section{Éditeur(s)}

Association Canadienne d'Ethnologie et de Folklore

ISSN

1481-5974 (imprimé)

1708-0401 (numérique)

Découvrir la revue

Citer cet article

Zaiane-Ghalia, S. (2016). Médiation culturelle pour la sauvegarde et la

valorisation du patrimoine tunisien : des initiatives citoyennes. Ethnologies,

38(1-2), 127-153. https://doi.org/10.7202/1041590ar

\section{Résumé de l'article}

Depuis son indépendance, la Tunisie a consacré des efforts importants à la valorisation du patrimoine culturel dans un objectif de développement économique misant sur le secteur touristique. Les diverses institutions gouvernementales concernées par la sauvegarde et la valorisation des richesses patrimoniales, telles que le ministère de la Culture et le ministère du Tourisme, ont mis en place plusieurs actions de médiation culturelle à cet effet. De nombreux rapports et publications mentionnent ces données mais l'on a peu écrit sur l'apport important des citoyens et des organisations communautaires à ce domaine de la médiation culturelle. Or le mouvement associatif a toujours été fort en Tunisie et il s'est renforcé depuis la révolution de 2010. À partir de visites et de rencontres personnelles effectuées sur le terrain et complétées par des données récentes colligées sur Internet - sur des blogues, des pages sociales ou des sites plus professionnels -, nous nous proposons d'examiner la place de la participation citoyenne dans la médiation culturelle en Tunisie. Nous prendrons à cet effet des exemples de projets concrets. 


\title{
Médiation Culturelle POUR la SAUVEGarde ET LA VALORISATION DU PATRIMOINE TUNISIEN
}

Des initiatives citoyennes

\author{
Selma Zaiane-Ghalia \\ Université de Moncton
}

\section{Introduction}

La médiation dans le domaine culturel englobe un ensemble de moyens d'information et de communication au service d'une interprétation variée et enrichie du patrimoine. Les options de cette médiation sont aussi vastes que l'est l'esprit de création, mais elles restent toutefois limitées par une logique politique et institutionnelle. Dans un contexte où la valorisation du patrimoine culturel passe nécessairement par l'évaluation économique des retombées, le tourisme culturel est l'option première visée. Toutefois, le processus tient compte aussi des volets de conservation et de valorisation de l'environnement, dans une perspective de durabilité. Dans cette optique, il est intéressant de voir le rôle joué par les citoyens et le mouvement associatif dans la médiation culturelle en Tunisie.

Depuis son indépendance en 1956, le gouvernement tunisien a investi des efforts importants en vue d'inclure la valorisation du patrimoine culturel dans une vision de développement économique misant sur le secteur touristique. Dans les années 90, des efforts additionnels ont été dévolus dans un cadre de développement durable. Cependant, cette attention à la protection des ressources patrimoniales avait déjà débuté par des efforts citoyens (Zaiane, 2004) et se prolonge encore de nos jours. Ainsi, il s'agit de la participation citoyenne. En effet, «Si bien que plus une communauté compte d'associations de bénévoles, plus elle est en bonne santé et ses membres se font davantage confiance et le sentiment d'appartenance à la communauté grandit et se traduit notamment dans une plus forte participation » (Benimmas, Boutouchent et Kamano, 2014 : 13). Or, selon ces mêmes auteurs, la participation citoyenne nécessite un sentiment 
d'appartenance articulé autour d'une identité collective qui se traduit dans des valeurs, des croyances et un héritage culturel et historique commun.

En témoigne la richesse des projets lancés par des citoyens dont nous allons présenter quelques exemples spécifiques que nous avons eu l'opportunité de visiter avant la révolution et dont nous sommes allés chercher l'évolution après la révolution, et ce, à travers des données réunies sur le web.

Dans son ouvrage traitant d'une éthique de la médiation, Jean Caune (1999 : 21) développe trois approches :

1- la médiation concerne les usages socio-politiques du terme, elle joue une fonction idéologique : elle apparaît comme un moyen que se donne l'institution pour maintenir le contact avec ses administrés et imposer des représentations et des relations sociales;

2- elle est d'ordre théorique et consiste à transformer la notion du sens commun en un instrument de pensée, c'est-à-dire un concept. Produire une signification partagée dans une communauté ;

3- elle apparaît comme un ensemble de pratiques sociales qui se développe dans des domaines institutionnels différents et qui visent à construire un espace déterminé et légitimé par les relations qui s'y manifestent (cité par Belhassine, $2010: 114$ )

Nous avons abordé ces trois approches à des degrés divers et à cet effet, nous avons entrepris de retracer sur le web, des projets et des organismes communautaires de promotion du patrimoine culturel. Lors de nos recherche sur le web, nous avons utilisé le moteur de recherche Google. Toutefois, nous avons croisé les données lorsque cela était possible pour vérifier l'authenticité des informations. Nous avons aussi visionné plusieurs vidéos d'entrevues avec les promoteurs des projets culturels. Nous nous sommes assurés de la crédibilité des sites retenus. Les critères de rigueur scientifique en recherche qualitative tels que les critères de crédibilité, de transférabilité, de fiabilité et de validité ont été appliqués tout au long de la démarche (Morse et al., 2002; Gohier, 2004).

Nous avons choisi des projets que nous avions visités avant la révolution et nous en avons retenu quatre majeurs qui puissent représenter la diversité culturelle. Nous avions eu l'opportunité, lors de nos visites au début des années 2000, de nous entretenir en personne avec chacun des promoteurs. Des entretiens individuels, semi-dirigés, non structurés, favorisant une écoute active de notre part, avaient eu lieu, ce qui permettait de mieux 
comprendre le sens qu'une personne donne à son expérience (Karsenti et Savoie-Zajc, 2000; Morissette, 2007).

Lors de ces entrevues libres nous avions réunis des informations sur le parcours de création du projet culturel et sur les difficultés rencontrées. Nous avions aussi recherché les motivations personnelles qui avaient poussé le promoteur à développer son projet culturel. Tous se sentaient alors comme « habité d'une mission de sauvegarde d'un patrimoine ».

Et, il est sans conteste que l'action citoyenne enrichit les projets de médiation mis en place par le gouvernement à divers niveaux hiérarchiques administratifs, mais permet aussi de combler des vides d'action. Nous avons analysé les progrès réalisés au niveau de ces projets de médiation culturelle lancés depuis les années 2000, tenant compte de l'évolution de la situation géopolitique du pays et des changements qui ont été apportés au niveau des institutions en charge de la sauvegarde et de la mise en valeur du patrimoine culturel. Nous avons par ailleurs identifié de nouvelles initiatives citoyennes et il est important de souligner la place importante des jeunes dans ce mouvement de développement de la médiation culturelle en Tunisie. Le succès des initiatives de développement communautaire menées par des jeunes après la révolution a été reconnu par divers rapports d'experts (Churchill, 2013; Banque mondiale, 2014 : 41; GIZ, divers projets de coopération municipale ${ }^{1}$ ).

La communication dans le domaine du patrimoine culturel est le lien entre, d'une part, la population locale et son patrimoine, et d'autre part, le touriste et le patrimoine national. Divers moyens de communication ont ainsi été utilisés afin d'attirer un large public tant international que national. Belhassine $(2010$ : 114) souligne que pour avoir lieu, la médiation requiert un dispositif technique ainsi que des acteurs inclus dans un contexte socio-culturel. La Tunisie a beaucoup investi pour s'adapter à un contexte mondial en évolution en matière d'exposition, d'offre muséographique et de médiation culturelle plus globalement (Ben Younès ${ }^{2}, 2009$ ). La valorisation des richesses patrimoniales reste depuis toujours fortement liée au développement touristique. Plusieurs institutions publiques et privées ont été impliquées dans cette démarche qui soutient un des piliers majeurs du développement économique du pays depuis son indépendance (Zaiane, $2004: 113)$.

1. http://www.co-mun.net/tunisie/promotion-des-jeunes/participation-des-jeunes. GIZ : Deutsche Gesellschaft für Internationale Zusammenarbeit.

2. Directeur de la division du développement muséographique au ministère de la Culture et de la Sauvegarde du patrimoine. 
La diversité des textes juridiques et des institutions concernées par le patrimoine démontre la complexité de mener à bien une politique de sauvegarde et de valorisation dans un paysage couvert par un riche patrimoine culturel très hétérogène, où une tradition de collaboration et d'approche interdisciplinaire trouve de la difficulté à se mettre en place. Le développement de la médiation culturelle est ainsi fortement lié à cette conjoncture, particulièrement en ce qui a trait à la liberté d'évolution de l'expression artistique citoyenne dans le cadre de la valorisation des richesses patrimoniales culturelles.

\section{Les partenaires institutionnels de la valorisation du patrimoine culturel}

La Loi 94-35 datée du 24 février 1994 désignée sous le nom de « Code du Patrimoine archéologique, historique et des arts traditionnels $»^{3}$, identifie les champs et les moyens d'intervention sur le patrimoine tangible. Cependant, l'élargissement considérable de la notion de patrimoine ces dernières années, amène à parler d'éclatement de cette notion (Audrerie, 2000 : 10). Les lois et règlements cherchent à s'adapter pour suivre cette évolution et tenir compte des orientations nouvelles. Depuis 2000, la législation a été enrichie en Tunisie, sans toutefois permettre d'améliorer la situation concernant la sauvegarde et la mise en valeur des richesses patrimoniales. La question de «qui fait quoi ? » reste posée (Abdelkafi, 2013).

Divers experts et chercheurs dénoncent le manque de coordination entre les différentes structures pour le bien de la sauvegarde et de la valorisation des richesses patrimoniales tunisiennes. Dans ce contexte, nous pouvons citer Habib Mellakh (2012), universitaire et syndicaliste du département de Français à l'Université de la Manouba, qui écrit que « les dangers qui menacent le patrimoine tunisien et les entraves spécifiques liées à sa gestion aux différents plans se rattachent à des problèmes structurels qui concernent la tutelle, la conception et la législation ». Il souligne l'absence de politique commune aux ministères de la Culture et du Tourisme. De son côté aussi, Jalel Abdelkafi (2013), urbaniste, a très souvent souligné cette absence de coordination entre les parties concernées par le patrimoine. Nous relevons aussi le témoignage de Mohamed-El Aziz Ben Achour, directeur général de l'ALECSO et ancien ministre de la culture et de la sauvegarde du patrimoine, dans sa déclaration en février 2011, lorsqu'il écrit parlant du village berbère de Chénini Tataouine :

3. http://www.intt.tn/upload/txts/fr/loi_36-1994-fr.pdf, texte de la Loi n 94-35 du 24 février 1994, relative au code du patrimoine archéologique, historique et des arts traditionnels, Journal Officiel de la République Tunisienne, 17 : 355-361. 
Il s'agit d'un des plus beaux villages de montagne de nos zones présahariennes. A mon initiative, l'INP a lancé un programme complet de protection, de sauvegarde et de restauration de ce bel exemple d'architecture vernaculaire. La mosquée des Sept dormants, située à proximité du village, superbe dans sa simplicité, a été sauvée in extremis d'une rénovation intempestive que voulaient y entreprendre le Ministère du tourisme et les Autorités régionales.

Référant international, l'UNESCO (2007 : 119) mentionne que les politiques culturelles des pays du Maghreb ont traversé plusieurs processus différents liés notamment aux formes d'organisations politiques et administratives adoptés par les gouvernements. En mars 2011, l'UNESCO a lancé un appel pour lutter contre les pillages des richesses culturelles dans les pays vivant la révolution (Shenu, 2011). Là encore, ce sont les mouvements citoyens qui vont, en Tunisie, se mobiliser pour la sauvegarde du patrimoine culturel et sa promotion (Meyer et al., 2012 ; Blaise, 2013). Aux organisations communautaires déjà en place, telles que les nombreuses ASM, Association de sauvegarde des médinas, ou encore l'Association tunisienne de sauvegarde des jeux et sports du patrimoine ${ }^{4}$, plusieurs nouvelles organisations ont vu le jour après la révolution. Nous mentionnons à titre d'exemples : l'Association Tunisienne pour la Sauvegarde des Musées et des Sites Archéologiques Tourath ${ }^{5}$ (2010); le réseau associatif Lam Echaml (2011); l'Association Twiza pour le Patrimoine, la Solidarité et le Développement (2011); l'Association Patrimoine 19-20 (2011), l'Association Tunisienne de la culture Amazigh (2011), l'Association de la documentation audiovisuelle du patrimoine tunisien (2011), ou encore, l'Association Actions Citoyennes en Médina (L'mdina Wel Rabtine, 2013).

Ces organismes à but non lucratif, ont bénéficié d'une révision de la législation en matière de création d'association (Décret-loi n² 2011-88 du 24 Septembre 2011 relatif aux associations). La législation en matière de

4. Créée par Ezzeddine Bouzid en 2006 avec comme objectif majeur sauvegarder et promouvoir les traditions culturelles ludiques. Parmi ces réalisations, l'association organise une formation sur la théâtralisation du conte traditionnel pour les jeunes animateurs le 27 mars 2014, à la maison de jeunes de Korba, ainsi que des jeux traditionnels de stratégies cognitives pratiqués par les personnes à besoins spécifiques, en février 2014.

5. Patrimoine est une association créée le 25 mai 2010 ayant pour objectif de contribuer à la préservation du patrimoine national et au développement du mécénat. Son président fondateur est Rejeb Elloumi. L'association a organisé un colloque International sur le « Mécénat » Culturel ayant pour thème « Patrimoine, Entreprises, Créativité » du 27 au 29 octobre 2010. 
conservation et de promotion du patrimoine a aussi souvent été modifiée. La diversité des textes juridiques relatifs au patrimoine tunisien ${ }^{6}$ et leur évolution dans le temps renforce la complexité des relations entre les différentes parties tant sur le plan national (les différents ministères : culture, tourisme, environnement, et autres), que sur le plan local (administrations d'une part, et citoyens de l'autre). Il ressort une profonde difficulté de définir la notion même de patrimoine et de responsabilités vis-à-vis de cette «Amana », cet héritage du passé, mais surtout, cet emprunt fait auprès des générations futures, car au même titre que la nature, le patrimoine culturel n'appartient pas à la génération présente, mais doit être sauvegardé et transmis aux générations futures. L'importance de la sensibilisation du public envers la valeur du patrimoine a toujours été un volet majeur pour les experts et les défenseurs du patrimoine (Larguèche, 2008) rien qu'à voir les nombreux séminaires qui sont organisés tant au niveau national, qu'au niveau international (par exemples : Biara, Ziani et Kadri, 2012 ; ou le projet Euro-Med Héritage $4^{7}$ ).

Pendant que les instances administratives s'organisent ou se réorganisent (Karamti, 2012; l'exemple de la situation des musées), les citoyens sont sur le terrain et mettent en place des projets concrets de médiation culturelle. Metoui et al. (2014) soulignent une «émancipation » de la société civile tunisienne. Le tissu associatif est « renouvelé $»^{8}$. Nous allons parcourir

6. Diverses institutions sont intéressées d'une façon directe ou indirecte par les potentialités économiques du patrimoine tant naturel que culturel du pays, qui est fortement convoité. Cela ressort au niveau de la complexité des rapports de collaboration et de l'instabilité des structures au vu des changements trop nombreux qu'ont subis certains ministères tels que le Ministère de la Culture (Décret $\mathrm{n}^{\circ}$ 2012-1885 du 11 septembre 2012, Journal Officiel de la République Tunisienne 75 : 2201-2203) anciennement, Ministère de la Culture et de la Sauvegarde du Patrimoine (Décret n 2005-1707 du 6 juin 2005). Alors qu'il avait été auparavant encore ministère de la culture, de la jeunesse et des loisirs (Décret $n^{\circ}$ 2004-760 du 15 mars 2004). Et préalablement en 2003, Ministère de la Culture aussi (Décret n² 2003-1819 du 25 août 2003).

7. Le projet associe sept partenaires actifs dans le domaine de la mobilisation de la société civile pour la conservation du patrimoine. Il est basé sur huit activités complémentaires dans lesquelles la participation des citoyens de la Méditerranée, et particulièrement des jeunes, est cruciale. http://www.euromedheritage.net/ intern.cfm? $\operatorname{lng}=$ fr\& menuID $=12 \&$ submenuID $=13 \&$ idproject $=44$

8. «Au cours des semaines qui suivirent la révolution, des dizaines de comités et d'associations ont été créés... On estime en effet que plus de 2000 nouvelles associations ont été créées depuis la révolution (Tainturier, 2012) ( Metoui et al. 2014 : 15). Selon une étude menée par la BAD auprès d'un échantillon de 250 associations, $43 \%$ œuvrent dans le domaine du développement communautaire 
quelques projets culturels citoyens : la médiation par la création de musées privés, la mise en place de site Web, de blogs, de pages Facebook et la valorisation d'autres médias de plus en plus accessibles avec les nouvelles technologies, mais aussi la médiation plus classique par des reconstitutions théâtrales de scènes de la vie quotidienne valorisant les traditions et les savoirs faire ancestraux, mais aussi par le développement spectaculaire de l'Art contemporain dans les rues et à la portée du grand public.

\section{Création de musées du patrimoine par les citoyens}

Pour certains historiens ou archéologues de l'Institut national du patrimoine, ces projets privés de musées ou écomusées du patrimoine traditionnel sont discutables. Ils ne respecteraient pas les normes de la muséographie selon les règles de l'ICOM. Mais peut-on reprocher à des citoyens de vouloir sauvegarder et mettre en valeur ce qu'ils considèrent comme des richesses patrimoniales de leur communauté locale ? Peut-on aussi reprocher à des citoyens d'être en avant-garde en matière de médiation, mais aussi de création d'un patrimoine culturel nouveau?

\section{Le Musée de la mer de Zarzis}

Nous l'avions visité en 2003. Le projet était à ses débuts, mais déjà bien identifié. Étant sur une propriété privée et ouvert gratuitement aux visiteurs, le projet n'a pas été empêché, mais les soutiens restaient absents. Pour son concepteur, Mohsen Lihidheb, il ne s'agit pas d'un hobby, d'un passe-temps, mais d'un travail de conviction. Chaque jour, il parcourt le rivage et ramasse les bouteilles. Parfois, il trouve des messages lancées à la mer. À travers des créations artistiques personnelles où chaque pièce retrouvée est mise en valeur, ce citoyen de Zarzis a su mettre en valeur la « culture de la mer ». Son œuvre a dépassé les frontières. En février 2002, il avait obtenu à Paris la reconnaissance du Guinness des records pour avoir réalisé « une collection de 26820 objets différents récupérés en 6 ans sur la plage de Zarzis ${ }^{9} »$. Et en 2014, il est mentionné dans l'ouvrage Museum Multiplicities : Field Actions and Research by Design, où les auteurs écrivent : «The images displayed in 'The Memory of the Sea' portray fragments of Mohsen Lihidheb's museum - The Sea Memory Museum in Zarzis, in

et $35 \%$ dans le domaine des arts et de la culture (Metoui et al. $2014: 17$ ).

9. Au début de 2013, il avait récolté plus de 700000 objets, parmi lesquels 54 bouteilles comportant des messages, dont il essaye toujours de retracer les messagers. http://www.repubblica.it/persone/2013/05/20/news/il_postino-59223839/. Entrevue filmée de l'artiste. 
Southern Tunisia, show what the sea has returned of unfortunate migrants who, set sail from the coast of Africa, have never landed on the European soil » (Basso Peressut et al., 2014 : 10). Cimoli présente ce musée comme un musée préhistorique éthnographique, soulignant qu'il « represents an artistic operation, and not just a work of preservation » (2014:36).

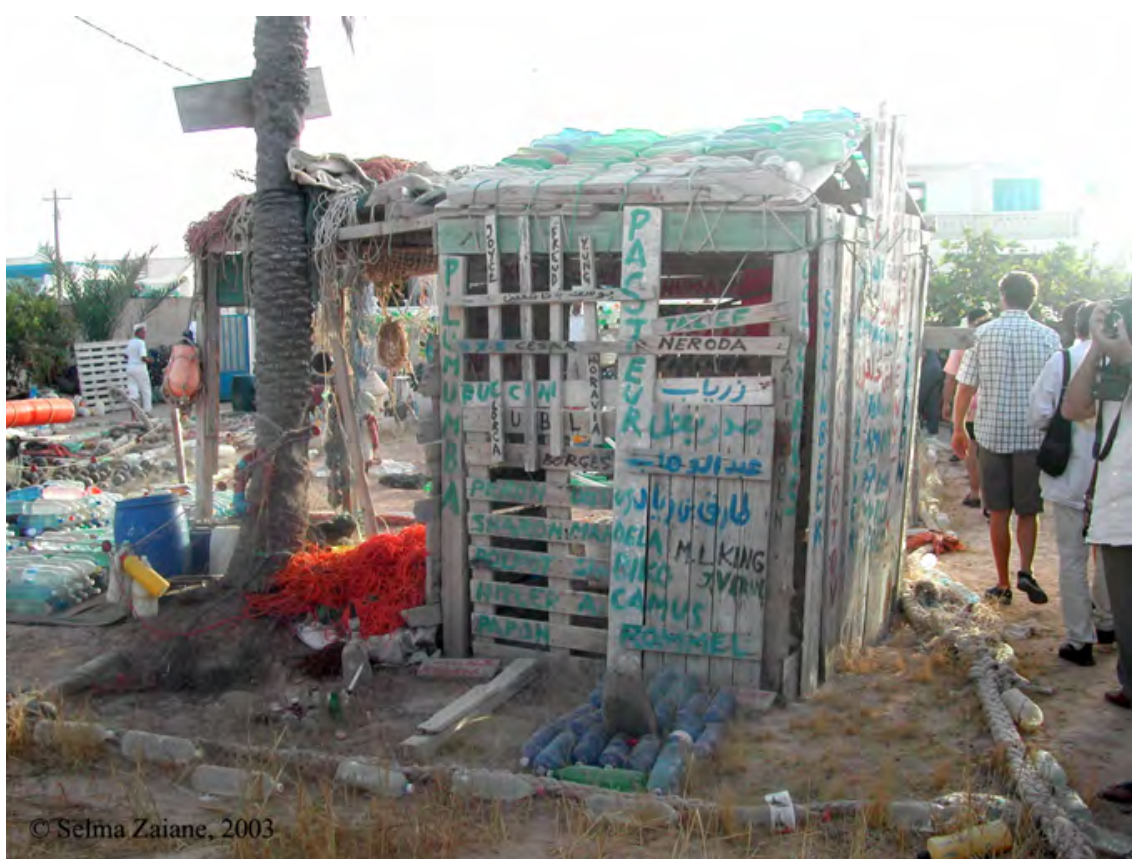

Photo 1 : Vue sur une partie du Musée de la Mer de Zarzis, 2003.

Sur le site des Artistes sans Frontières ${ }^{10}$, l'artiste présente plusieurs de ses œuvres, parfois éphémères, créées sur le bord de l'eau à la portée du public et appelant à la contemplation méditative, à la réflexion. Mohsen Lihidheb est aussi un poète. Et à travers ses textes, qui sont aussi un support de médiation culturelle non négligeable, il communique sa passion de la mer et de la nature, mais aussi de l'importance de la restauration du patrimoine. Voici un extrait du poème « Poterie » :

Je tourne toujours autour du pot,

Parce que je le vois cassé,

Et ce n'est pas forcement mon lot,

Car tout le monde doit y penser,

Sauver la terre, sauver la vie,

10. http://art.artistes-sf.org/mohsen/ 
Sauver ces belles poteries,

Est une urgente restauration,

Pour colmater les fissions,

Lihidheb mohsen

Zarzis Tunisie 03/01/04

\section{la Repubblica.j Persone}

Home $\mid$ Politica
Persone Arte $\mid$ Biglietti di eventi| Passaparola

$$
\text { G+1 } 11 \text { Twest } 23 \text { G consligla } 834
$$

\section{Mohsen, il postino del Mediterraneo}

A Zarzis, un villaggio tunisino che a periodi si sovraffolla di gente pronta a partire su barconi pericolanti in direzione dell'Europs, l'incontro $\infty$ n questo personaggio. Un giorno consegnava posta, oggi va all'alba a racoogliere quello che il mare ridà alla terra. E lo conserva in un museo a cielo aperto II KAMI FARES

\section{Lo leggo dopo}

(20 maggio 2023) \& Riproduzione riservata

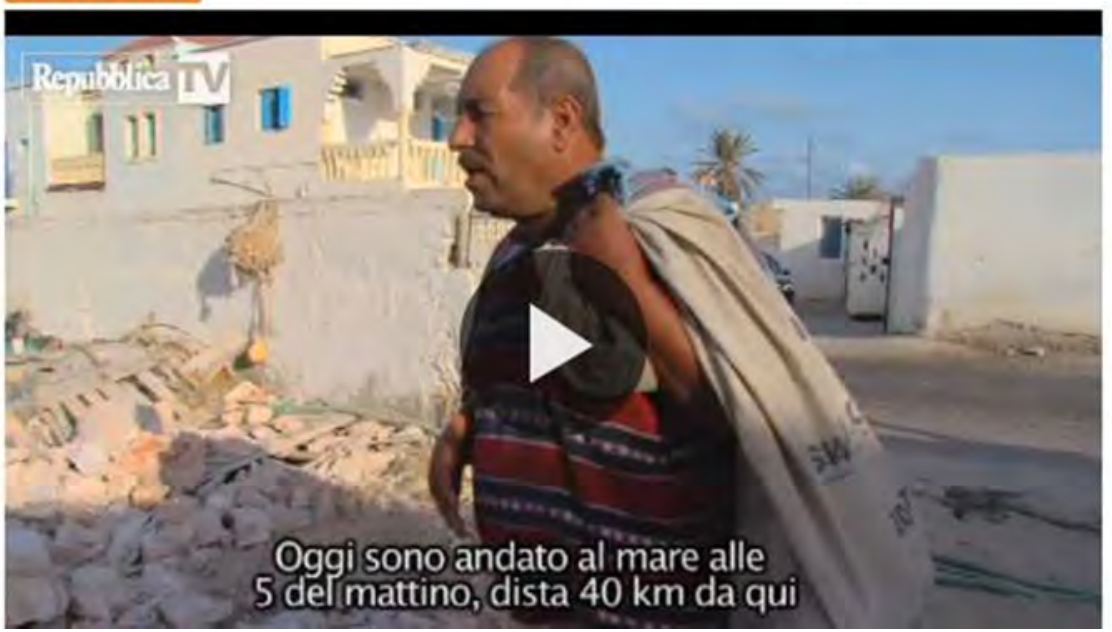

Photo 2 : Capture écran de l'article sur le site web de La Republica.it, le 28/10/2015 
Ces créations d'art ont été promues sur le plan international par la réalisation d'un film documentaire de $26 \mathrm{mn}$, «Sacrées bouteilles », écrit et réalisé par Fitouri Belhiba, support de médiation culturelle important, qui présente l'histoire de ce musée original ${ }^{11}$. Ce film a été suivi par un autre en 2013, "Il postino del Mediterraneo », réalisé par Kamikairy Fares et Giulia Ardizzone, dont on peut voir un extrait ${ }^{12}$ avec l'artiste présentant son parcours. Cet extrait a été partagé via Facebook plus de 834 fois et twitté par 23 personnes, à la date du 28 octobre 2015. Ce qui indique la force de ce média audiovisuel dans la médiation culturelle et l'apport citoyen important pour la promotion de Zarzis et de la culture de la mer.

\section{L'écomusée du Rocher Bleu}

Située sur un promontoire rocheux qui a inspiré à sa promotrice le nom du lieu touristique et culturel « Le Rocher Bleu ${ }^{13}$ », ce musée fait partie d'un plus large projet de relais culturel et de restauration : «Espace Culturel Jaouida Guiga », situé au village berbère de Takrouna dans la délégation d'Enfidha, gouvernorat de Sousse. La propriétaire le mentionne comme étant l'écomusée de Dar Gmach centre d'art et de tradition populaire. Toutefois cette appellation d'écomusée reste complexe car elle est utilisée, en Tunisie, spécifiquement au niveau des espaces naturels. Mais ceci ne semble pas poser d'obstacle pour la mise en valeur des artéfacts patrimoniaux qui ont été réunis et présentés d'une façon spontanée, sans étude muséographique spécifique préalable. Cependant, Aida Bellagha Gmach, la promotrice a toujours été ouverte aux suggestions des experts qui lui ont rendu visite. Ses efforts sont reconnus et elle le dit « des muséographes du Musée Naturel d'Aix en Provence m'ont félicitée pour la simplicité et l'homogénéité du musée »(Djait Amel, 2009). La médiation culturelle mise en place par Madame Gmach lui a demandé beaucoup d'énergie, de patience et de persévérance pour réussir à convaincre les différentes parties concernées par la sauvegarde de ce village antique berbère. Lorsqu'elle dit : " J'ai réussi à faire de ce village fantôme, qui n'était plus que l'ombre de lui-même à un moment, un point de rencontres » (Djait Amel, 2009), c'est une réalité, pour tous ceux qui connaissent le lieu avant le projet et aujourd'hui. La médiation peut-être ici soulignée au niveau de la définition

11. http://zarzissea.skyrock.com/

12. http://www.repubblica.it/persone/2013/05/20/news/il_postino-59223839/, mis en ligne le 20 mai 2013.

13. Le nom a aussi été donné en hommage à l'écrivain Tahar Guigua, originaire du village, en référence à son recueil de nouvelles, Le Grand rocher. (Djait Amel, 2009). 
du terme en tant que l'intermédiaire, le médiateur, entre le patrimoine et la population : dans le sens où la promotrice a réalisé plusieurs actions de protection du site en réveillant le sentiment de fierté des habitants afin de les faire participer à cette sauvegarde du site. À cet effet, elle raconte que « il a fallu aussi que je me fasse accepter par les villageois. Bien que je sois originaire de Takrouna, je suis une intruse, étrangère à leur système » (Djait Amel, 2009). Mais elle a aussi joué le rôle de médiatrice auprès des autorités responsables des infrastructures et du patrimoine, afin que celles-ci soient présentes et remplissent leurs rôles. Elle dit à cet effet que « obtenir les autorisations de l'Institut National du Patrimoine (INP) a été titanesque » (Djait Amel, 2009). Et la médiation culturelle est aussi réalisée par Madame Gmach à travers les photos, vidéos et autres informations qu'elle partage aux visiteurs ${ }^{14}$. Plusieurs autres sites font mention de ce projet culturel et des activités d'animation que Madame Gmach organise pour la promotion du site et du village de Takrouna : Florence Batisse-Pichet (2012) écrit «Vous serez accueilli chaleureusement par une hôtesse charmante, artiste peintre et céramiste, Aïda Gmach Bellagha, à l'origine de cet écomusée original qu'elle a monté avec passion sans compter ses heures. Une tunisienne qui incarne le sens de l'hospitalité », ou encore Isabelle Enault (2011) qui mentionne « L'héritière de la famille Gmach garante du patrimoine culturel ... un écomusée ‘Dar Gmach' qui renferme une collection privée des plus riches et des plus diversifiées de l'artiste peintre Ali Bellagha ».

Ce projet est devenu si médiatique, qu'on le retrouve mentionné par plusieurs personnes et sur plusieurs sites web. Ces supports virtuels deviennent les meilleurs médiateurs et promoteurs sur le plan marketing. Nous pouvons mentionner l'exemple de Gérald di Giovanni, concepteur de l'Académie Orientaliste ${ }^{15}$, lequel en réponse à la question «Pensez-vous que le tourisme soit encore un moyen de communication et de rapprochement entre les cultures et les peuples? », répond:

14. Sur son blog «Le Rocher bleu au village berbère de Takrouna » : http://takrouna. blogs-de-voyage.fr/ ). Ce blog est même mentionné comme référence par le Guide touristique Petit Futé Tunisie de 2009, dirigé par Jean-Paul Labourdette, p.240 et 243 et sa page Facebook du groupe privé Le Rocher Bleu au village Berbère de Takrouna qui compte 506 membres (le 28 octobre 2015). Il est à noter que le fait que la page Facebook soit privée ne permet pas à tout le monde d'en voir le contenu et il faut donc que l'internaute fasse l'effort de faire la demande d'adhérer au groupe. Ce qui en limite la portée comme support de médiation culturel.

15. Une association qui favorise les échanges culturels et le savoir-faire pictural entre les des deux rives de la Méditerranée. Entrevue du 28 mai 2010, http://www.milleet-une-tunisie.com/accueil/rencontres/687-gerald-di-giovanni-1-un-bel-exemplepour-decouvrir-lautre-tunisie-r.html. 
Je ne préconise pas le transat et la serviette de plage comme mode de tourisme absolu. Bien au contraire... A quelques kilomètres des plages de Hammamet, il existe un petit village juché sur un promontoire rocheux que j'affectionne particulièrement: Takrouna! Et cette Tunisie-là, doit aussi être proposée (quoique je garderais volontiers l'endroit secret!). J'y emmène mes stagiaires toujours avec plaisir parce que ce lieu surprend. Il suscite souvent de belles émotions par et pour son authenticité. Voilà un bel exemple de découverte d'une autre Tunisie.

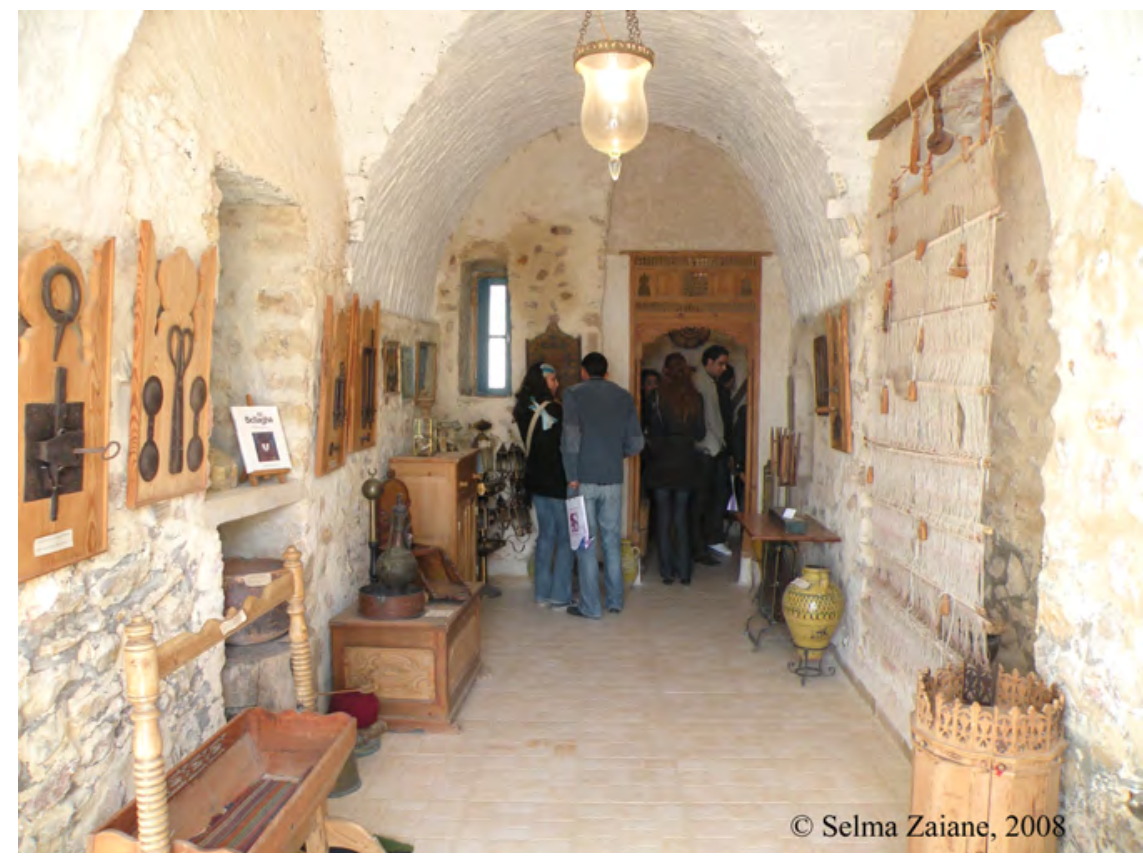

Photo 3 : Entrée de l'écomusée 'Dar Gmach' au sein de l'espace culturel Le rocher Bleu à Takrouna, 2008.

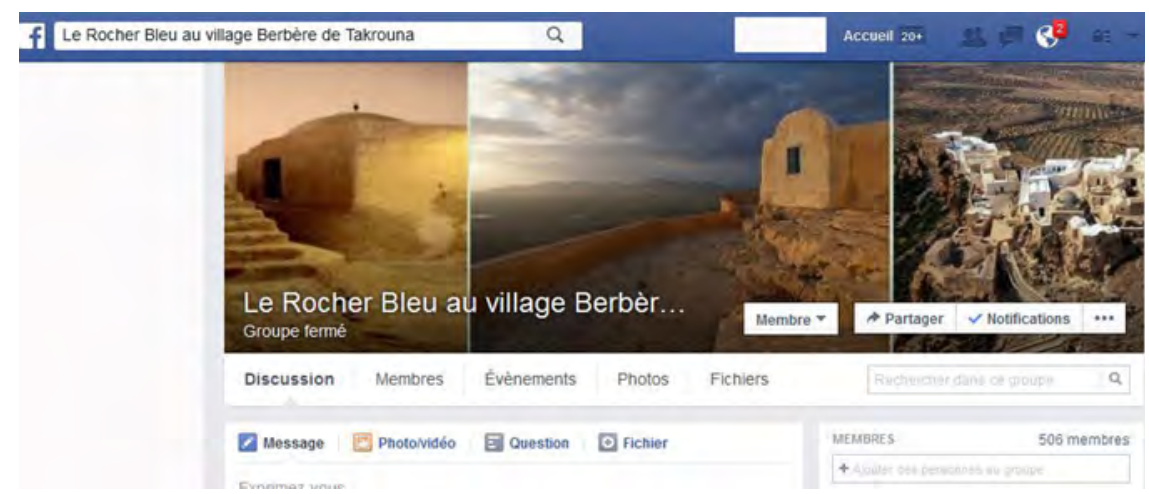

Photo 4 : Capture écran de la page Facebook privé du Rocher bleu, 506 membres à la date du 28 octobre 2015. 
Nous ne pouvons faire abstraction de l'aspect culturel gastronomique du restaurant du Rocher Bleu. En effet, Madame Bellagha, avec la complicité de son mari, a mis au point une recette du pain «Mlaoui », qui ravi toute personne qui en fait l'expérience. Et un bloggeur a voulu partager son expérience en prenant des photos des étapes de la préparation de ce pain (la recette restant secrète) $)^{16}$.

\section{L'art contemporain à la sauvegarde de la Médina de Tunis : Dream City}

Plusieurs projets étaient lancés au moment de la révolution qu'a vécue la Tunisie fin 2010. Qu'en sera-t-il de l'avenir du patrimoine culturel tunisien lorsqu'on lit sur internet que « les menaces sur le patrimoine de la médina de Tunis se précisent » (Bourial, Hatem, 2013) ? Doit-on orienter différemment les projets de médiation culturelle pour la survie des richesses patrimoniales du pays afin d'éviter leur destruction ? Comment une nouvelle médiation culturelle pourrait-elle aider à sauvegarder le patrimoine ? Bien des questions restent posées, néanmoins l'action citoyenne est en place. Et c'est depuis 2007 que deux jeunes artistes chorégraphes, Selma et Sofiane Ouissi, que nous avons eu l'occasion de rencontrer à diverses reprises en 2010, ont lancé le projet ambitieux de la Dream City Biennale d'Art Contemporain sur l'espace public en Tunisie. Ce concept de médiation culturelle, Hamza Alya (2010) le qualifie de « chasse à la culture ", dans le sens que c'est l'éveil du grand public de 7 à 77 ans à l'appréciation de nos richesses patrimoniales dans leur plus large diversité, allant du patrimoine bâti au patrimoine immatériel des traditions et des savoirs faire. Pour Selma et Sofiane Ouissi, il s'agit d'amener le public à "rêver et se réapproprier la Médina de Tunis », non seulement sur le plan spatial, mais aussi sur le plan temporel, mettant l'accent sur l'histoire porteuse de mémoire (2012, Éditorial). Ils soulignent que « cet 'art in progress' a pour horizon une réappropriation de la ville par un exercice inédit de la citoyenneté tunisienne et du droit à l'exercice de la liberté pour participer au développement d'une culture démocratique » (2012, Éditorial). En octobre 2015, leur page Facebook comptait plus de 12829 «j'aime $»^{17}$.

16. Blog de Voyage, « Le voyage de Philippe », il écrit « Le Rocher Bleu de Takrouna, outre son paysage, son histoire, son panoramique exceptionnel, offre aussi des instants uniques d'amitiè et de partage qu'il est difficile de narrer sans un brin de nostalgie! », http://latunisiedemesreves.blogs-de-voyage.fr/2008/09/01/cours-decuisine-au-rocher-bleu-de-takrouna/.

17 Le site web officiel du projet culturel est : http://www.lartrue.com/fr/index 


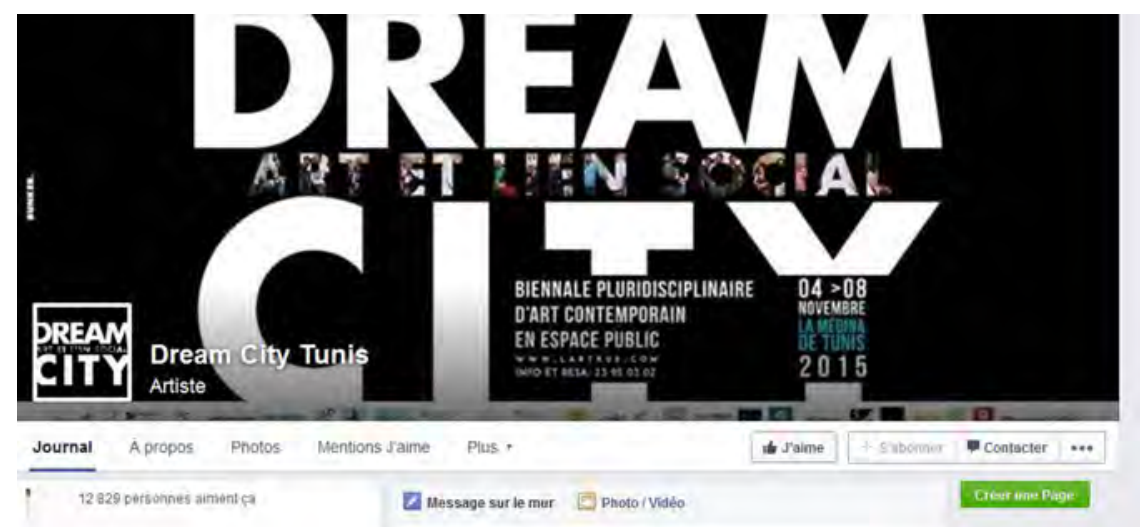

Photo 5 : Capture écran de la page Facebook de Dream city, le 28/10/2015

Dream City, en investissant plusieurs lieux de la Médina, a peut-être ouvert les portes à d'autres initiatives citoyennes pour la valorisation de la culture de la Médina de Tunis. Ainsi, l'association Pontes Tunisie (créée en décembre 2011) a organisé le 1er mars 2015, dans le cadre de son projet «Reviving Bab Souika : la culture facteur de développement, citoyenneté et engagement », une journée autour de la «Tijâniyya », une des expressions de la spiritualité populaire tunisienne ${ }^{18}$. Et nous mentionnons aussi le projet d'inventaire et protection des hammams historiques de la médina de Tunis

Association Actions Citoyennes en Médina (L'mdina Wel Rabtine)

Iseptembere of

Sute de raffaire Dar Djait: "La balle serait alors dans le camp municipal pour son manque de vigilance et sa protection élastique du patrimoine, en fonction des interlocuteurs " hittp / / Www webdo.tr. . /un-collectif-citoyendenonce-un-mass 1

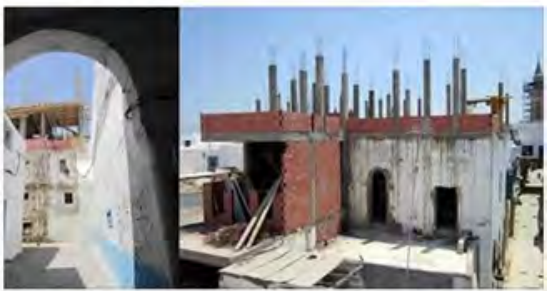

Un collectif citoyen dénonce un "massacre" urbain dans la médina de Tunis

Dernières actualités de Tunisie avec réquipe de webdo

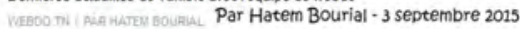

Photo 6 : Capture écran sur la page FB de l'association Actions citoyennes en Médina. lancé par l'Association Actions Citoyennes en Médina (Fournet Thibeaud et Redon Bérangère, 2013). Cette organisation compte plus de 2582 membres sur sa page Facebook le 28 octobre 2015. Elle utilise dans le cadre de sa médiation culturelle, divers médias sociaux. En plus de la page Facebook et de la mise en ligne de ses rapports d'activités, elle utilise les blogs, tel que inkyfada.com tenu par Blaise Lilia et Sbouai Sana (2014) qui ont réalisé un reportage illustré de très belles photos et d'une carte de localisation des hammams historiques dans la Médina. Sur sa

18. http://www.cultart.tn/decouvrir-tijaniya-musique-histoire-traditions/ et http:// www.pontestunisie.net/ 
page $\mathrm{FB}$ cette organisation partage aussi divers articles qui appellent à la participation citoyenne pour la sauvegarde du patrimoine culturel de la Médina de Tunis (exemple photo 6). Encore un support de médiation culturelle original issue d'une initiative citoyenne.

\section{Le patrimoine de Gafsa : L'ASM un pilier de médiation culturelle}

Sans une réelle gouvernance locale, il devient difficile d'assurer la mise en place d'une médiation culturelle réussie et durable, car un des acteurs principaux est alors absent, à savoir : la population locale. En l'état actuel des choses, la gouvernance patrimoniale est aléatoire. Or nous voyons avec l'exemple de Gafsa et les efforts importants fournis par les membres de l'Association de sauvegarde de la Médina (ASM) de Gafsa $^{19}$, l'importance cruciale de cet acteur pour assurer la sauvegarde des richesses patrimoniales et leur mise en valeur. Cette association milite

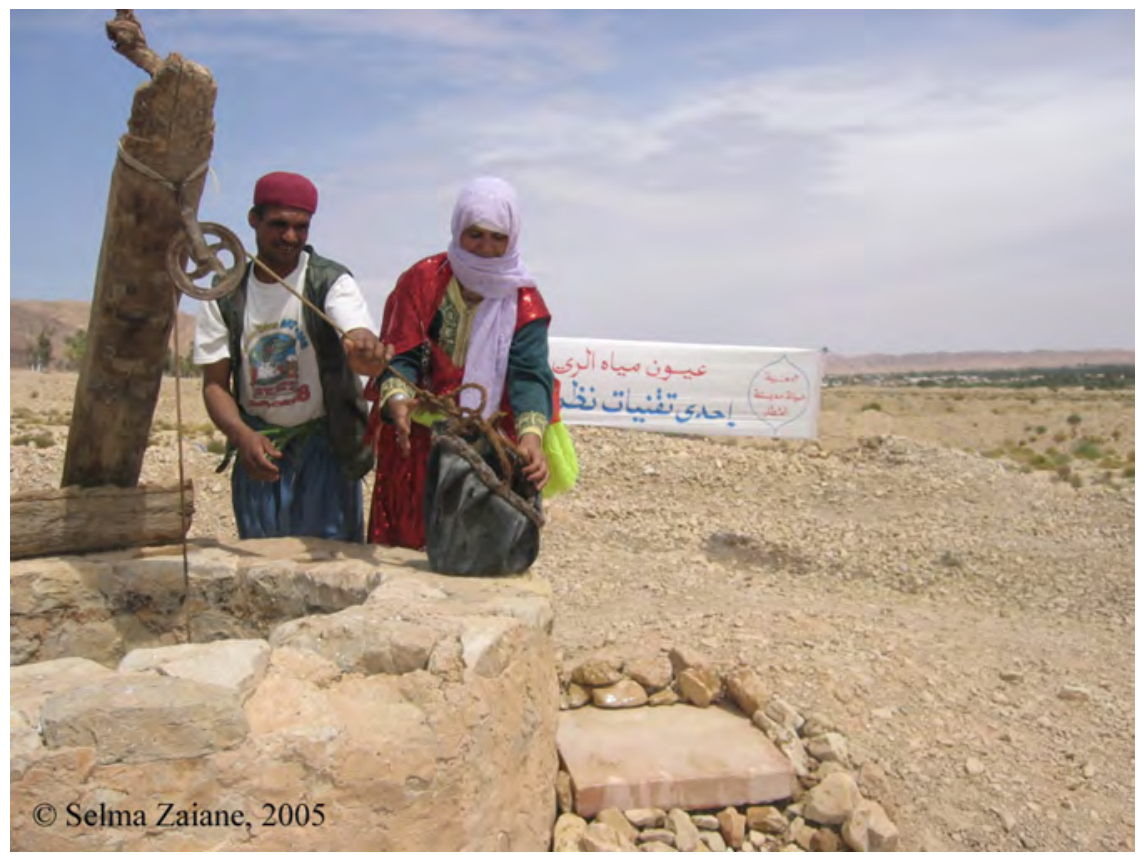

Photo 7 : Reconstitution d'une scène de puisage d'eau dans un des puits à El Guettar, 2005

19. Voir sur la page Facebook de l'Association de Sauvegarde de la Médina de Gafsa et sur le site internet de l'ASM, une présentation de leurs réalisations en vue de sauvegarder et de valoriser non seulement la médina, mais toute les régions limitrophes dont font partie les oasis qui renferment un riche patrimoine naturelle, mais aussi un patrimoine immatériel irremplaçable au niveau des savoir faires. 


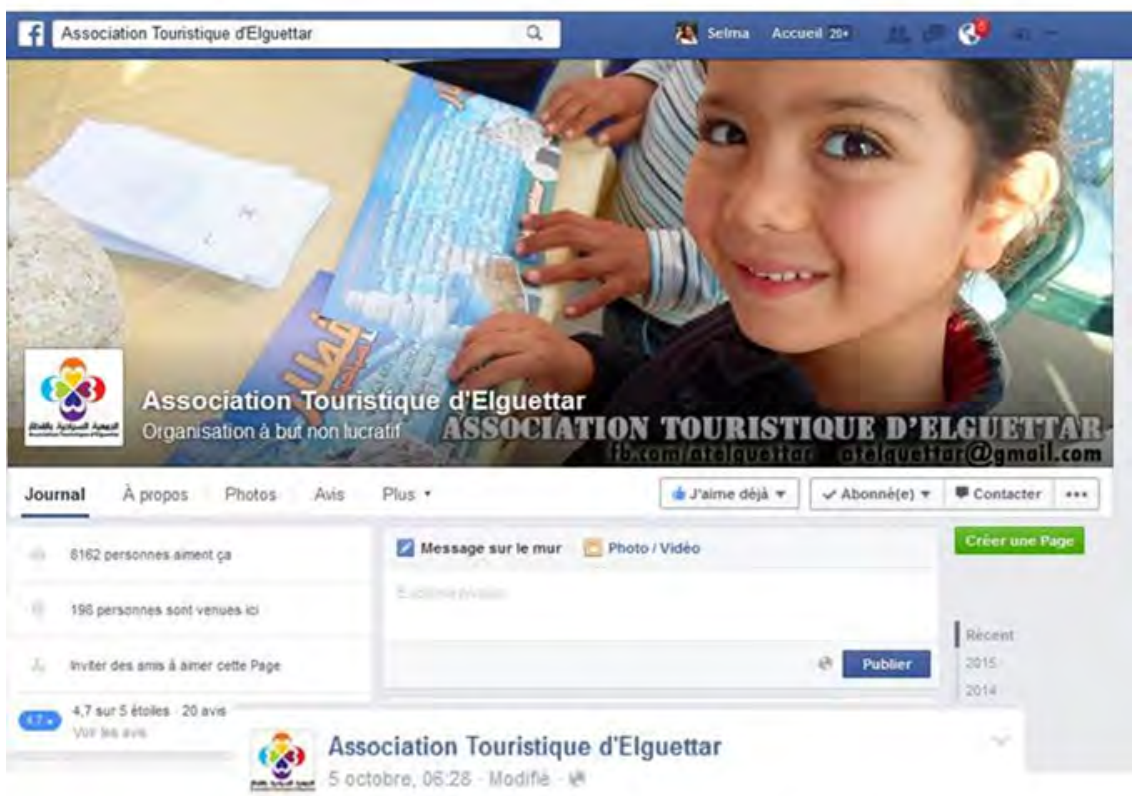

L'Association Touristique d'Elguettar, organise sans le cadre de son projet "PalmTour", un cycle de formation "Guide Touristique Local" pour former une vingtaine de guides touristiques locaux basés à Elguettar. II s'agit d'une formation qui sera encadrée par des experts en tourisme alternatif et accompagnement touristique. La formation est par alternance et comprend des visites du circuit touristique d'Elguettar. Un certificat ATE sera délivé à la fin du cycle de formation aux participants qui auront suivi assidûment l'ensemble des 5 sessions et réussi lexamen final.

Cette formation s'adresse prioritairement aux jeunes diplômés motivés d'Elguettar voulant s'impliquer dans la dynamique touristique de la région.

Les candidats intéressés doivent envoyer au plus tard le 31 Octobre 2015 un CV et une lettre de motivation (en arabe ou en français) à l'adresse de TATE :

E-mail : atelguettar@gmail.com en mettant comme Object « Formation - Guide Locals.

W'aime Commenter $A$ Partager

Photo 8 : Captures écran sur la page FB de l'association touristique d'El Guettar, 28 octobre 2015

sur tous les aspects patrimoniaux : de la sauvegarde des savoir-faire de la culture oasienne agricole, aux bâtis de la médina de Gafsa, en passant par les traditions locales, et dépassant les limites directes de la ville pour soutenir des projets tel que la remise en état de la série de puits antique dans la région d'El Guettar en périphérie de l'Oasis de Gafsa. Et en septembre 
2012, une association spécifique au développement touristique d'El guetta a vu le jour. L'Association touristique d'El Guettar comptait près de 2900 membres sur sa page Facebook en mars 2015 et 8162 le 28 octobre 2015, soit 5262 membres de plus en 7 mois. Elle organise même une formation pour des guides touristiques locaux (voir photo 8 ).

Dans le cadre de ses diverses activités de médiation culturelle, l'ASM de Gafsa participe à l'organisation de festivals au cours desquels des reconstitutions de scènes de la vie quotidienne sont présentées au public (Photo 7), des photos anciennes sont reproduites et partagées pour permettre au grand public de mieux connaitre le patrimoine de la région, mais aussi pour donner l'occasion aux habitants de Gafsa et aux jeunes en particulier, de se réapproprier leur histoire et d'en être fiers, les sensibilisant ainsi à sa sauvegarde ${ }^{20}$.

En 2013, L'ASM a publié un bel ouvrage intitulé « Gafsa, une terre, une histoire, des hommes ». On retrouve la promotion de cet œuvre sur plusieurs sites Internet, dont un mentionne ceci :

En achetant le livre proposé au prix de 65 Dinars Tunisiens (40 Euros / 50 dollars américains - frais d'envoi postal non inclus), vous contribuerez à acquérir et aménager une «ghaba» dans l'oasis historique de Gafsa pour en faire un conservatoire de la biodiversité et un musée vivant des outils et techniques de l'agriculture oasienne. Un projet qui permettra sans nul doute de valoriser notre patrimoine oasien et à faire de Gafsa un pôle touristique et culturel..$^{21}$

Cet ouvrage et sa promotion via Internet nous montrent le mariage réussi des supports de communication et de promotion plus anciens, tel les livres, et des supports plus modernes offerts par les nouvelles technologies de la communication et le Web 2.0. Ce projet de livre réalisé par l'ASM n'est qu'un des multiples projets de promotion et de développement culturels mis en place par l'équipe de bénévoles de l'ASM guidée par leur président Lazhar Chérif. Ce dernier est très actif et multiplie ses présentations sur les potentiels patrimoniaux de la région de Gafsa ${ }^{22}$. Il faut dire que la personne même de Lazhar Chérif est devenue un « support de médiation culturelle », tant il incarne la région de Gafsa et est rapidement reconnu

20. L'importance de l'image dans la médiation culturelle a été longuement analysée par Belhassine Sarra (2010), qui a abordé les Ksours du Sud-Est tunisien.

21. http://www.hammam-ensa.com/details_articles.php?art_id=2348\&cat_id=44, consulté le 6 mars 2013.

22. Exemple d'une récente présentation faite à Rome, en Italie, en avril 2014, sur le Système de l'oasis historique de Gafsa, http://www.fao.org/fileadmin/templates/ giahs/Presentations/april2014sc/02/_FN_Tunisia.pdf 
par de nombreuses personnes.

\section{L'usage des supports technologiques pour la médiation culturelle citoyenne}

Nous avons mentionné quelques exemples précédemment. Ces exemples ne couvrent pas la large diversité de projets de médiation culturelle qui se sont renforcés ou ont pu éclore suite à la révolution en Tunisie. Ils ont permis à un amour de la sauvegarde et de la valorisation du patrimoine, trop longtemps étouffé, de pouvoir éclore au grand jour et de s'épanouir. Le site CulturArt - Agenda culturel ${ }^{23}$, permet de voir la grande diversité des activités culturelles présentes au niveau de la capitale, Tunis. Leur page Facebook, ouverte le 18 juillet 2014 avec le sous-titre de « Votre agitateur culturel à Tunis », comprenait 19428 membres à la date du 27 octobre 2015.

L'impact de la coopération internationale citoyenne dans le cheminement vers la sauvegarde et la promotion du patrimoine tunisien ne peut être omis. "Initiative citoyenne » englobe tout citoyen du monde. Ainsi, nous retrouvons plusieurs amoureux et passionnées de la Tunisie, qu'ils soient tunisiens expatriés ou étrangers nés en Tunisie, et bien d'autres profils. Nombreux sont les gens à travers le globe qui admirent la richesse historique et le patrimoine culturel de la Tunisie et, pour cette raison, se mobilisent pour les faire connaitre. A titre d'exemple nous pouvons mentionner le site de Christian Jung ${ }^{24}$, un franco-canadien né en Tunisie. Le site est principalement dédié à la région de Bizerte où Chrisitan Jung a vécu plusieurs années, mais il promeut aussi d'autres régions ainsi que les musées et les sites archéologiques. Ce site a été visité par plus de 1224692 internautes (IP) ${ }^{25}$ depuis sa création à la fin de 2001, et le 15 juin 2014 plus de 346 personnes se sont inscrites à la newletter en une seule journée. Le site a reçu en une semaine 910 visiteurs (2-8 mars 2015) et depuis mai 2007, 20696011 pages du site ont été visionnées.

Les défenseurs du patrimoine sont ainsi toujours actifs. Les citoyens qui croient fermement dans la valeur tant culturelle qu'économique des riches patrimoniales de la Tunisie, continuent à mettre toute leur énergie en commun afin de les protéger d'une destruction potentielle. Une rage de destruction synonyme d'ignorance et d'aliénation individuelle ou collective, s'exerce parfois sur des monuments qui ont traversé le temps, à l'exemple

23. http://www.cultart.tn/

24. http://lac-de-bizerte.com/oye.html

25. À la date du 27 octobre 2015 . 
des divers marabouts, monument de Saints, qui ont été profanés ${ }^{26}$ (Zaiane Selma, 2012). Mais là encore l'action citoyenne pour la préservation a répondu présente. A l'exemple de la reconstruction du mausolée de Sidi Bou Saïd (Blaise Lilia, 2013).

Ainsi, il ne fait aucun doute que la société civile est bien consciente de la nécessité de sauvegarder le patrimoine culturel qui est la richesse du pays et son pilier d'avenir à travers le développement d'activité de loisir culturel et du tourisme. Slah Smaoui, architecte, artisan et promoteur du projet touristique Village Ken, écrit ceci sur sa page Facebook :

Aucun politique ne défend un Projet Culturel, alors que la Tunisie en a besoin d'urgence si l'on aspire à un vrai développement harmonieux de notre Pays... La société doit s'organiser indépendamment des politiques, et chacun dans son domaine si elle veut sauver la Tunisie... (30 décembre 2013).

Comme le web aurait donné naissance à la révolution tunisienne (Lecomte Romain, 2011), sera-t-il aussi au service de la médiation pour la sauvegarde et la promotion de son patrimoine ? Cela nous parait bien possible juste à voir le nombre croissant de blogueurs ${ }^{27}$ qui présentent les richesses du pays, les associations de sauvegarde des médinas qui font connaitre leurs activités sur le web, ou encore le Magazine MagÉco qui a publié un numéro spécial sur le tourisme culturel ( $\mathrm{n}^{0} 3$ du 15 mars 2012) et qui comptait le 28 octobre 2015, 22612 membres sur sa page Facebook. Et encore le magazine "Saisons tunisiennes », éditée par Skandrani Raja, qui compte 691 membres sur sa page privée Facebook ${ }^{28}$ à la même date, et dont l'objectif est de promouvoir la nature, la culture et le patrimoine touristique tunisiens. C'est dans un esprit d'engagement citoyen qu'il a lancé ce magazine, accessible gratuitement sur le web.

Le choix du thème de la troisième session d'Orange Mobility Forum qui s'est déroulé le 6 décembre 2012 à Tunis, n'a pas été retenu par hasard. Son message de promotion indiquait que « À l'heure du tout numérique,

26. On pouvait lire dans un des articles qui rapportent les divers incidents que «Après le mausolée de Sidi Abdelaziz à la Marsa, en banlieue nord de Tunis, c'est le mausolée de Sidi Bou Saïd qui a été incendié dans la nuit du samedi 12 janvier. La population a protesté dans les rues de la banlieue nord pour manifester sa colère face à la recrudescence de ces attaques contre le patrimoine culturel. » (Blaise Lilia et Boufaied Amine, 2013).

27. Exemple du blog 'Nouveau tourisme culturel' : http://www.nouveautourismeculturel. com/blog/2012/04/25/tourisme-culturel-en-tunisie/, consulté le 31 décembre 2013.

Ou la page Facebook de Vacances chez l'habitant Tunisie.

28. Le 7 mars 2015. 
comment les jeunes développeurs peuvent contribuer à la promotion du tourisme culturel tunisien en concevant des applications innovantes pour téléphones mobiles et autres tablettes ${ }^{29}$. La finalité de ce forum aura été de «sensibiliser les parties prenantes ainsi que le public à l'intérêt de ce mélange qui réunit le tourisme et la technologie pour valoriser le patrimoine culturel » (Achour Ali, 2012). Aussi, selon le directeur général d'Orange Tunisie, Didier Charvet, « 44\% des voyageurs ont téléchargé une application sur le tourisme pendant leur voyage » (Achour Ali, 2012). Nous allons certainement voir un renforcement dans le développement d'applications permettant de promouvoir et de valoriser le patrimoine culturel tunisien à travers les nouvelles technologies de la communication numérique. Ceci est même déjà une réalité. Le musée national du bardo offre la possibilité d'une visite audio-guidée depuis 2010, mais aussi la visite en 3D sur le site du musée ${ }^{30}$ et ceci depuis 2012. Plus récemment, en juin 2014, grâce à un partenariat entre la compagnie privée Orange et le ministère de la culture, un guide de visite numérique du musée a été lancé. Ce guide, agrémenté par des jeux, permet une découverte ludique des œuvres les plus importantes.

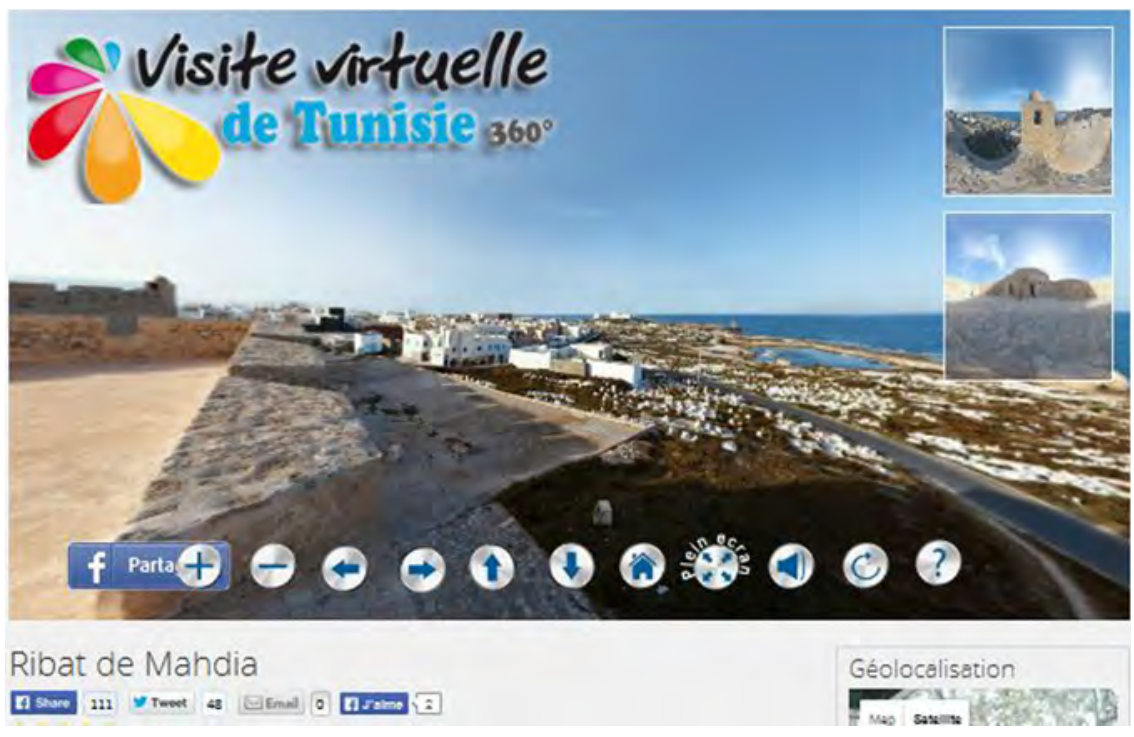

Photo 9: Capture écran de la visite virtuelle en $360^{\circ}$ du Ribat de Mahdia, 28 octobre 2015

29. News (sans auteur), Quelle applications innovantes pour soutenir le tourisme culturel en Tunisie, publié le 3 décembre 2012, http://www.leaders.com.tn/ article/quelles-applications-innovantes-pour-soutenir-le-tourisme-culturel-entunisie?id=10022, consulté le 31 décembre 2013.

30. http://www.bardomuseum.tn/index.php\%3Foption\%3 Dcom_ content\%26view\%3Darticle\%26id\%3D280\%26Itemid\%3D97 
Conçu avec un format d'application pour Smartphone, il est téléchargeable gratuitement sur Google Play, l'App-Shop d'Orange Tunisie ${ }^{31}$.

Et pour reprendre l'implication citoyenne plus directe, nous pouvons mentionner l'exemple de la visite virtuelle du Ribat de Mahdia offerte par le photographe Sami Frikha ${ }^{32}$, partagé plus de 110 fois et tweetée par plus de 48 personnes, à la date du 28 octobre 2015.

Il y a aussi via internet un accès à des films, des documentaires, des reportages, reconnus depuis toujours comme des supports de médiation culturelle. Mais il y a aussi des émissions radiophoniques sur le Web. Et ces radios prennent de plus en plus une place de choix dans la diffusion de messages de promotion culturelle. Nozha Smathi (2010:59) souligne que «Internet présente aux artistes les moyens d'affirmer leur indépendance vis-à-vis de la tutelle culturelle en l'absence de l'appui des médias locaux ».

\section{Conclusion}

Suivant une démarche d'analyse de cas, nous avons tenté d'apporter plus de lumière sur la diversité de l'action associative et citoyenne qui est de plus en plus le cœur et les poumons de la médiation culturelle pour la sauvegarde et la valorisation du patrimoine en Tunisie. Après la révolution dite du «printemps arabe » ou du « jasmin », la promotion du patrimoine culturel tunisien a gagné une grande part sur la place publique. L'expression artistique et la médiation culturelle s'est enrichie, libérée, développée, épanouie, et elle surprend à chaque jour le tunisien, mais surtout l'étranger qui ne connaissait peut-être pas encore la créativité sans fin des jeunes tunisiennes et tunisiens et leur passion pour leur patrimoine.

Reprenant Chaumier et Mairesse (2013), nous dirons que la médiation est une mise en relation ou un vecteur de transmission de contenus à des fins d'enrichissement interpersonnel. Ainsi la médiation culturelle virtuelle rendue possible grâce aux nouvelles technologies, à commencer par le web 2.0., est le point de contact, le déclenchement, qui crée des circonstances d'un rassemblement autour d'une cause ou d'un projet culturel pour les tunisiens.

Chaumier et Mairesse (2013) soulignent que «Si l'individu est visé par le processus de médiation - mise en culture -, c'est plus largement son ancrage dans un collectif et dans une communauté d'appartenance qui est

31. http://www.orange.com/fr/actualites/2014/juin/musee-national-du-BardoTunis

32. http://www.vvt360.com/divers/archeologie/visite-virtuelle-ribat-mahdia 
sollicité ». C'est ce que nous avons vu naitre et croitre depuis la révolution et qui a permis une libération de la créativité et de l'expression culturelle en Tunisie, soutenue par une large utilisation des supports informatiques par la jeunesse tunisienne, mais pas seulement, car nous retrouvons sur internet des étrangers 'amis ou amoureux du patrimoine culturel tunisien', qui participent pleinement au mouvement citoyen.

En provoquant chez l'individu une réflexion ou un engagement, on produit un investissement d'ordre culturel qui jette des ponts entre intériorité personnelle et construction solidaire. Cette vision constitue un projet politique qui s'incarne dans le renouvellement des formes culturelles, plus engageantes, interactives et participatives (Chaumier et Mairesse, 2013).

Nous avons constaté l'impact de plus en plus grandissant de la promotion touristique via les blogs personnels de voyageurs (Morelli Pierre, 2009 ; Maguer Amandine, 2011), au point qu'en septembre 2014, le premier salon des blogueurs de voyages francophones a été organisé à Cannes, en France (Khlat, Mathilde, 2014 et Emily, 2014) et le Zéme édition se tiendra en mai 2015 à Ajaccio $^{33}$.

Aussi, l'éventail de projets culturels tunisiens que nous avons présenté ici ne couvre nullement la réalité de la diversité et de la richesse des actions citoyennes en faveur de la médiation culturelle. Nous avons analysé un échantillon de ressources identifiées sur le web. C'est un domaine qu'il faudrait approfondir et dont l'État tunisien doit tenir compte au niveau des soutiens économiques à prévoir d'une part, mais surtout, au niveau de la gestion du volet de la médiation culturelle. Sachant la place indéniable que tient le tourisme pour la survie de l'économie du pays, il n'est plus de choix que de reconnaitre la place qui va de droit au citoyen dans l'effort de reprise économique du secteur, en soutenant les jeunes et moins jeunes promoteurs dans leurs efforts de médiation culturelle sur le web, à travers leurs pages Facebook, leurs blogs, les nombreuses photos et vidéos partagées et toute autre forme de support informatique qui permet de diffuser l'information culturelle au-delà des frontières du pays.

Nous avons mentionné quelques exemples de citoyens qui sont "médiateurs culturels ». Ils sont eux-mêmes, en tant que personne, devenus les représentants de leur région, du patrimoine culturel qu'ils défendent et veulent mettre en valeur. L'État tunisien devrait reconnaitre publiquement leurs efforts et leurs décerner un prix «Le prix de la médiation culturelle citoyenne».

33. http://salon-blogueurs-voyage.com/ 


\section{Références}

Abdelkafi, Jellal, 2013, « Portée et limites du programme Euromed Heritage, sur le patrimoine culturel de la Méditerranée ». Conférence Régionale pour la clôture du programme Euromed Heritage 4 : http:// www.euromedheritage.net/euroshared/doc/Jellal\%20ABDELKAFI_ Portee\%20et\%20Perspectives_FR.pdf

Achour, Ali, 2012, «mTourisme : Quand Orange s'intéresse à la promotion du tourisme en Tunisie », Tunisie Haut Débit, 10 décembre 2012 : http:// www.thd.tn/index.php?option $=$ com_content $\&$ view $=$ article\& $\mathrm{id}=2272$ :mtourisme-quand-orange-sinteresse-a-la-promotion-du-tourisme-entunisie\& catid $=56:$ fai\& Itemid $=50$

Association Actions Citoyennes en Médina, 2014. "Inventaire et protection des hammams historiques de la médina de Tunis. Rapport de la table ronde », Balaneia, thermes et hammams, 12 juillet 2014 : https://balneorient.hypotheses.org/2776

Audrerie, Dominique, 2000, La protection du patrimoine culturel dans les pays francophones. Paris, Editions Estem.

Banque mondiale, 2014, Tunisie: Surmonter les barrières à l'Inclusion des Jeunes. Rapport No. 89233-TN. Washington, Banque Mondiale.

Basso Peressut, Luca, Cristina F. Colombo et Gennaro Postiglione, 2014, Museum Multiplicities: Field Actions and Research by Design. Milan, MELA Books.

Batisse-Pichet, Florence, 2012, "Le Rocher Bleu : l'écomusée de Takrouna », Côté Maison, 6 septembre 2012, http://blogs.cotemaison. $\mathrm{fr} /$ chemin-lisant/2012/09/06/takrouna-village-berbere/.

Belhassine, Sarra, 2010, Penser la médiation culturelle au Sud-Est tunisien : Objets et stratégies de valorisation. Thèse de doctorat en cotutelle internationale en sciences de l'information et de la communication. Université Paul Verlaine-Metz. École doctorale Perspectives interculturelles: écrits, médias, espaces, sociétés. France; et Université de la Manouba, Institut de presse et des sciences de l'information. Tunisie.

Ben Achour, Mohamed-El Aziz, 2011, "Déclassements de terrains à Carthage et Sidi Bou Said: l'ancien ministre Mohamed-El Aziz Ben Achour s'explique ». Leaders, 23 février 2011 : http://www.leaders.com. tn/article/4232/print

Benimmas, Aïcha, Fadila Boutouchent et Lamine Kamano, 2014, « Le sentiment d'appartenance chez les parents d'élèves immigrants francophones du Nouveau-Brunswick ». Canadian Ethnic Studies 41(1): 87-119. 
Ben Younès, Habib, 2009, « Tunisie: Développement muséographique - Un impératif : l'actualisation des textes juridiques ». La Presse de Tunisie, 16 janvier 2009.

Biara, Ratiba Wided, Abdel Wahab Ziani et Soria Kadri, 2012, « La sensibilisation du grand public: une mission, au fondement du lien entre le passé et l'avenir d'une société ». Dans Actes de la deuxième Conférence régionale euro-méditerranéenne. Sous le thème "La ville, patrimoine vivant ", Sale, Maroc 11 et 12 octobre 2012 : 183-190. Barcelone, Montada.

Blaise, Lilia, 2013, «En Tunisie, le mausolée de Sidi Bou Saïd fait peau neuve grâce à la mobilisation citoyenne ». Harissa.com, 29 juillet 2015 : http://www.harissa.com/news/article/en-tunisie-le-mausol\%C3\%A9ede-sidi-bou-sa\%C3\%AFd-fait-peau-neuve-gr\%C3\%A2ce-\%C3\%A0la-mobilisation-citoyenne.

Blaise, Lilia et Amine Boufaied, 2013, «Tunisie - Deux mausolées incendiés pendant le week-end». Slate Afrique, 14 janvier 2013 : http://blog. slateafrique.com/tawa-fi-tunis/2013/01/14/tunisie-\%E2\%80\%93-deuxmausolees-incendies-pendant-le-week-end/

Blaise, Lilia et Sana Sbouai, 2014, «Hammam, patrimoine oublié. En Tunisie les hammams traditionnels tombent dans l'oubli. L'absence d'entretien et la perte de la pratique les excluent lentement de la mémoire patrimoniale ». Inkyfada.com, 29 septembre 2014 : https:// inkyfada.com/2014/09/hammam-patrimoine-tradition-culture-medinatunisie/

Bourial, Hatem, 2013, « Les menaces sur le patrimoine de la médina de Tunis se précisent ». Webdo.tn, 9 janvier 2013 : http://www.webdo. tn/2013/01/09/les-menaces-sur-le-patrimoine-de-la-medina-de-tunisse-precisent/

Caune, Jean, 1999, Pour une éthique de la médiation. Le sens des pratiques culturelles. Grenoble, Presses universitaires de Grenoble.

Chaumier, Serge et Françoise Mairesse, 2013, La médiation culturelle. Paris, Armand Colin.

Churchill, Erik, 2013, Le travail de jeunesse en Tunisie après la révolution. Paris, Programme Euromed Jeunesse IV.

Cimoli, Anna Chiara, 2014, «The Memory of the sea : Exhibiting a Meseum». Dans Luca Basso Peressut, Cristina F. Colombo et Gennaro Postiglione (dir.). Museum Multiplicities: Field Actions and Research by Design : 36-51. Milan, MELA.Bokks.

Djait, Amel, 2009, «Takrouna avec Aida Bellagaha Gmach : Le Rocher bleu est un défi. Mon combat est de préserver notre patrimoine », 
nacerboudjou.over-blog.com, 31 août 2009 : http://nacerboudjou.overblog.com/article-35477224.html

Emily, 2014, «Premier salon des blogueurs de voyage francophones ». Travel and film, 14 août 2014 : http://travelandfilm.com/premier-salon-desblogueurs-de-voyage-francophones/

Enault, Isabelle, 2011, « Takrouna, l'authenticité berbère à une heure de Tunis ». Au Féminin Canada, 12 mai 2011 : http://www.aufeminin. com/guide-voyage/takrouna-village-berbere-de-takrouna-entunisie-s734212.html

Fournet, Thibeaud et Redon Bérangère, 2013, « Sauvegarde des hammams historiques de la médina de Tunis. Mobilisation citoyenne pour la sauvegarde durable des hammams historiques de la médina de Tunis ", Balaneia, thermes et hammams, 22 octobre 2013 : http://balneorient. hypotheses.org/2634

Gohier, Christiane, 2004, "De la démarcation entre critères d'ordre scientifique et d'ordre éthique en recherche interprétative ». Recherches qualitatives $24: 3-17$.

Hamza, Alya, 2010, « Spectacle de rue. Dream City, rêver la ville, rêver la vie ». La Presse de Tunisie, 20 juillet 2010 : http://www.lapresse. $\mathrm{tn} / 08022015 / 9423 /$ dream-city-rever-la-ville-rever-la-vie.html

Karamti Yassine, 2012, "Le musée dans le contexte du Printemps arabe : le cas de la Tunisie ». France Diplomatie : http://www.diplomatie.gouv. fr/fr/IMG/pdf/musees_2012_karamti_01_cle0c523c.pdf

Karsenti, Thierry et Lorraine Savoie-Zajc, 2000, Introduction à la recherche en éducation. Sherbrooke, Éditions du CRP.

Khlat, Mathilde, 2014, « Les blogueurs voyage, des «influenceurs» convoités par le tourisme. La blogosphère s'est professionnalisée ». i-Tourisme, 30 octobre 2014 : http://www.tourmag.com/Les-blogueurs-voyage-desinfluenceurs-convoites-par-le-tourisme_a70179.html.

Larguèche, Abdelhamid, 2008, «L'Histoire à l'épreuve du patrimoine ». L'Année du Maghreb 4 : 191-200.

Lecomte Romain, 2011, «Révolution tunisienne et Internet : le rôle des médias sociaux ». L'Année du Maghreb 7 : 389-418.

Maguer, Amandine. 2011, Comment les nouvelles technologies valorisent elles le séjour touristique?. Master 1, Aménagement, urbanisme et développement touristiques durables. Université Michel de Montaigne, Bordeaux 3.

Mellakh, Habib, 2012, «Appel pour la création d'un Conseil National du Patrimoine tunisien ", Leaders, 16 août 2012 : http://www.leaders.com. tn/article/appel-pour-la-creation-d-un-conseil-national-du-patrimoine- 
tunisien?id=9093

Metoui, Mokhtar, Ahmed Mainsi, Henda Gafsi et Carmen Malena, 2014, La société civile dans une Tunisie en mutation. Rapport pour le PNUD. http:// touensa.org/wp-content/uploads/2014/07/La-soci\%C3\%A9t\%C3\%A9civile-dans-une-Tunisie-en-mutation.pdf

Meyer, Vincent, Mongi Sghaïer et Nozha Smati (dir.), 2012, «Développement territorial, patrimoine et tourisme en zones fragiles et menacées : entre dynamiques économiques, démocratie participative et communication ». Revue des Régions Arides 28 (2) : http://f.hypotheses. org/wp-content/blogs.dir/1155/files/2012/12/Actes-du-colloque.pdf

Morelli, Pierre, 2009, «Blog et tourisme. L'exemple de la Tunisie». Dans Dynamiques de développement : au carrefour des mondes : actes du $4 e$ colloque EUTIC : 629-639. Lisbonne, Centro de Investigacoa para Tecnologias Interactivas.

Morissette, Karine, 2007, "L'apport des sources orales à l'étude d'une situation particulière de communication interculturelle : l'interprétariat ». Recherches qualitatives Hors-Série 4 : 90-102.

Morse, Janice M., Michael Barrett, Maria Mayan, Karin Olson et Jude Spiers, 2002, «Verification Strategies for Establishing Reliability and Validity in Qualitative Research ». International Journal of Qualitative Methods 1 (2) : 13-22.

Ouissi, Selma et Sofiane Ouissi, 2012, «Itinéraires Art contemporain Espace public Tunisie. L'artiste face aux libertés ». Dossier de presse de la $3^{\mathrm{e}}$ Biennale d'art contemporain en espace public DREAM CITY. Tunis 26-30 septembre 2012, Sfax 5-7 octobre 2012.

Shenu, Isabelle, 2011, "L'Unesco alerte sur le patrimoine culturel en Tunisie, Egypte et Libye ». RFI Afrique, 25 mars 2011 : http://www. rfi.fr/afrique/20110325-unesco-alerte-le-patrimoine-culturel-tunisieegypte-libye.

Smati, Nozha, 2010, «Constructions Radiophoniques de la Culture en Tunisie: Quelles Médiations?». ESSACHESS Journal for Communication Studies 3(6) : 49-61.

Tainturier, Pierre, 2012. «Le bouillonnement de la société civile ». Magasine Faim et développement, $263:$ http://ccfd-terresolidaire.org/fdm/2012/263janvier-fevrier/les-defis-d-une-toute

Unesco, 2007, Les politiques culturelles au Maghreb. Paris: Unesco. http:// www.un.org.ma/IMG/pdf/unesco_clt_04_fr.pdf.

Zaiane, Selma, 2004, Tourisme et loisirs dans les parcs nationaux tunisiens. L'exemple du parc national d'Ichkeul. Tunis, Centre de Publication Universitaire. 
Zaiane-Ghalia, Selma, 2012, «Les routes des marabouts : des lieux de recueillements transfrontaliers au sein des parcs nationaux tunisiens ». Dans Laurent Bourdeau, Pascale Marcotte et Mohamed Habib Saidi (dir.), Actes du Colloque international : Routes touristiques et itinéraires culturels, entre mémoire et développement. Québec (Canada) du 13 au 15 juin 2012, organisé par l'université Laval: 479-490. Québec, Presses de l’Université Laval.

Sites internet

Association de la documentation audiovisuelle du patrimoine tunisien. http:// bac-associations.tn/portrait-de-lassociation-de-la-documentationaudiovisuelle-du-patrimoine-tunisien/; http://sara-dahdouh.com/_ adapt/?page_id=2

Association de Sauvegarde de la Médina de Gafsa. http://www.asmgafsa. org.tn/sipam_oasis_gafsa/index.html

Association Tourath. http://www.association-tourath.org/

Association Tunisienne de la culture Amazigh. https://www.youtube.com/ watch?v=7QERbevWbFg

Association Patrimoine Dix-neuf/Vingt. http://www.archi-mag.com/ actu_286.php, 17 septembre 2011.

Réseau des associations citoyennes LAM ECHAML. http://registration. fsm2015.org/view_orga/1759; http://www.lamechaml.org/

\section{Pages Facebook}

Association Actions Citoyennes en Médina (L'mdina Wel Rabtine)

Association de Sauvegarde de la Médina de Gafsa

Association Tourath

Association tunisienne de sauvegarde des jeux et sports du patrimoine.

Association Twiza pour le Patrimoine, la Solidarité et le Développement 